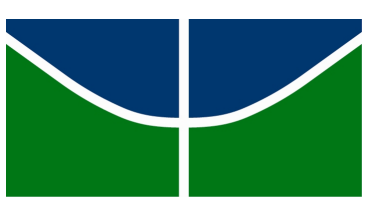

Universidade de Brasília - UnB

FACE - Faculdade de Administração, Contabilidade e Economia

Departamento de Economia - Programa de Pós-Graduação

\title{
Escolha Sob Categorias
}

Aluno: Bruno de Albuquerque Furtado

Orientador: Leandro Nascimento, PhD

Co-Orientador: Gil Riella, $\mathrm{PhD}$ 
Universidade de Brasília - UnB

FACE - Faculdade de Administração, Contabilidade e Economia

Departamento de Economia - Programa de Pós-Graduação

\section{Escolha Sob Categorias}

Dissertação apresentada ao Departamento de Economia como requisito para obtenção do título de Mestre em Ciências Econômicas.

Aluno: Bruno de Albuquerque Furtado Orientador: Leandro Nascimento, $\mathrm{PhD}$ Co-Orientador: Gil Riella, PhD 


\section{Agradecimentos}

A Adolfo e Marta, meus pais, que sempre me revelaram os melhores caminhos, dentre os quais o mestrado em economia.

A meus amigos de vários anos, em especial Danilo, meu irmão, por sempre estarem dispostos a discutir sobre qualquer assunto, mesmo (principalmente?) os mais herméticos.

Aos professores Gil Riella e Leandro Nascimento, pela orientação, sem a qual esta dissertação nunca teria passado de uma conjectura.

Aos professores José Guilherme de Lara Resende, Rodrigo Peñaloza e Daniel Cajueiro, por sempre proporcionarem aos alunos a oportunidade de expandir seus conjuntos de possibilidades de criação.

Aos colegas do programa de pós-graduação em economia, especialmente João Vítor Rego Costa e Caio Guimarães Figueiredo, por ajudarem a tornar o indecifrável complicado e o complicado trivial.

À Juliana, cuja graça, inteligência e coragem, eternamente crescentes, jamais cessam de me surpreender e inspirar. 



\section{Resumo}

Propomos um modelo de escolha racional na presença de categorias. Dada uma categorização subjetiva do conjunto de alternativas inteiro, o agente, ao deparar-se com um problema de escolha, seleciona os melhores elementos disponíveis de cada categoria. O modelo explica certos desvios importantes do Axioma Fraco da Preferência Revelada, mas é plenamente caracterizado por outras propriedades observáveis do comportamento de escolha do agente. No caso mais geral, nossa representação generaliza a maximização de preferências incompletas. No caso particular em que as categorias são disjuntas, provamos que ela equivale à maximização de preferências incompletas acrescida de uma propriedade bastante intuitiva.

Palavras-chave: categorias. racionalidade limitada.

Classificação JEL: D01 



\section{Abstract}

We propose a model of rational choice in the presence of categories. Given a subjective categorization of the choice set, the agent, when faced with a choice problem, picks the best elements available from each category. The model explains certain important deviations from the Weak Axiom of Revealed Preference, while being fully characterized by other observable properties of the agent's choice behaviour. In the more general framework, our representation generalizes the maximization of incomplete preferences. For the specific case in which categories are disjoint, we prove that it is equivalent to the maximization of incomplete preferences plus a somewhat intuitive property.

Keywords: categories. bounded rationality.

JEL classification: D01 



\section{Sumário}

Sumário . . . . . . . . . . . . . . . . . . 1

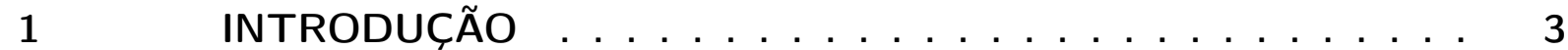

2 DEFINIÇÕES PRELIMINARES . . . . . . . . . . . . . 5

$2.1 \quad$ Escolha e comportamento observável . . . . . . . . . . . . . 5

$2.2 \quad$ Preferências e categorias . . . . . . . . . . . . . . . . 6

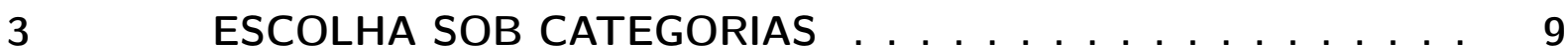

$3.1 \quad \mathrm{O}$ caso geral $\ldots \ldots \ldots \ldots \ldots$

$3.2 \quad$ Categorias disjuntas . . . . . . . . . . . . . . . . . . 12

$4 \quad$ CONCLUSÃO $\ldots \ldots \ldots \ldots \ldots$

REFERÊNCIAS . . . . . . . . . . . . . . . 21 



\section{Introdução}

O processo de categorização é um dos procedimentos cognitivos mais fundamentais e prevalentes. Sua importância jaz no fato de que ele nos permite entender e fazer previsões quanto a objetos e eventos em nosso mundo (MEDIN; AGUILAR, 1999; SMITH; MEDIN, 1981), bem como escolher dentre uma gama, por vezes enorme, de alternativas disponíveis. Quando uma pessoa deseja adquirir um plano de telefonia celular, o mais provável é que ela não considerará toda a gama de produtos, organizará em ordem decrescente de preferência, para aí então escolher seu favorito. Ela primeiramente vai categorizar os planos de acordo com certas características - como a qualidade e tamanho dos pacotes de dados, se eles são pré-pagos ou pós-pagos, a faixa de preço, e por aí em diante -, para apenas então escolher os melhores produtos de cada categoria.

De fato, uma crítica recorrente à teoria da escolha racional é a de que ela não leva em consideração o papel da categorização nos processos decisórios de seres humanos. ${ }^{1}$ Desenvolvemos, portanto, um modelo que se afasta da estrutura tradicional da escolha racional precisamente no sentido em que ele leva em consideração categorizações.

Presumimos que o tomador de decisões é dotado de preferências incompletas relativas a todo o espectro de alternativas possíveis das quais ele pode ser intimado a escolher. Por "incompletas" queremos dizer que há pares de alternativas no espectro de escolhas que não podem ser gradadas como melhores, piores ou indiferentes uma em relação a outra. Para uma dada categorização do espectro de escolhas, o agente só compara elementos dentro de categorias, e então escolhe os melhores dentro de cada uma. A escolha final do agente será simplesmente a união, sobre todas as categorias, dos elementos escolhidos dentro das categorias. A única restrição que será imposta sobre a categorização é que as preferências dentro de cada categoria sejam completas.

É um fato bem estabelecido na teoria da decisão que se racionalidade completa é compreendida como o 'comportamento que é compatível com a maximização de preferências transitivas e completas', então ela é equivalente a 'comportamento que satisfaz o Axioma Fraco de Preferências Reveladas de Samuelson (Weak Axiom of Revealed Preferences WARP)'. A despeito de sua aparente simplicidade, o modelo apresentado neste artigo dá conta de muitas violações importantes do WARP.

Por exemplo, se algum tomador de decisões escolhe a opção x na presença tanto de y quanto de $\mathrm{z}$ em escolhas binárias, mas em seguida nunca escolhe $\mathrm{x}$ quando todas as alternativas, $\mathrm{x}, \mathrm{y}$ e $\mathrm{z}$, estão disponíveis simultaneamente, ele está exibindo uma forma de dependência de menu (menu dependence), que é um tipo fundamental de violação de WARP. $^{2}$ De fato, um padrão de comportamento deste tipo não pode nem mesmo ser

1 Para um exemplo de uma crítica deste tipo, ver Schwartz (2000).

2 Para ver uma decomposição de WARP em dois tipos de consistência, uma das quais é independência 
representado pela maximização de preferências incompletas, conforme caracterizado por Eliaz e Ok (2006) e Riella e Ribeiro (2015). Acontece, no entanto, que a representação por categorias exposta nesta dissertação é capaz de descrever acuradamente este tipo de comportamento não ortodoxo de escolha.

Recentemente, muitos enfraquecimentos de WARP foram propostos na literatura, levando a diversos modelos de 'racionalidade limitada'. O que mais se assemelha a nosso trabalho é Manzini e Mariotti (2012). O modelo destes, chamado Categorize-DepoisEscolha (Categorize-Then-Choose - CTC) consiste em um processo de duas etapas: na primeira etapa, o agente classifica todas as categorias dentre o espectro factível, eliminando todas as alternativas pertencentes a uma categoria dominada; na segunda etapa, ele escolhe sua alternativa preferida dentre aquelas restantes. A partir disto, a diferença crucial entre o CTC e o nosso modelo é que o CTC assume que o agente é capaz de fazer comparações entre categorias, bem como dentro delas. Já foi sugerido na literatura que escolhas entre categorias podem ser problemáticas, ${ }^{3}$ e portanto restringimos nosso modelo a escolhas dentro de categorias. Nenhuma prioridade é dada para uma categoria sobre qualquer outra.

Na seção 2, apresentaremos alguns conceitos chave, incluindo a definição de categorização. A seção 3 descreve e interpreta os resultados. Finalmente, na seção 4 estão algumas considerações finais, incluindo sugestões para estudos futuros.

de menus, ver Manzini e Mariotti (2012).

3 Schwartz (2000, pp. 82): "Within each category, it may be relatively easy to express preferences. Between categories, however, expressing preferences is more problematic." 


\section{Definições Preliminares}

\subsection{Escolha e comportamento observável}

Seja $X$ o conjunto arbitrário não-vazio que representa todas as alternativas mutuamente exclusivas disponíveis a um tomador de decisões. Chamamos uma classe $\Omega_{X} \subseteq 2^{X} \backslash\{\varnothing\}$ de um campo de escolha em $X$. Um dado campo de escolha em $X$ é interpretado como a classe de todos os problemas de escolha que o tomador de decisões é capaz de confrontar, e portanto chamamos qualquer $A \in \Omega_{X}$ de um problema de escolha. No momento, precisamos apenas que $\Omega_{X}$ inclua todos os conjuntos unitários de $X$ e seja fechado sob uniões finitas. Mais estrutura pode e será imposta sobre $\Omega_{X}$ conforme necessário. Nós nos referimos a qualquer par $\left(X, \Omega_{X}\right)$ em que $|X| \geq 3$ e $\Omega_{X}$ é um campo de escolha em $X$ como um espaço de escolha. Se $\Omega_{X}$ é a classe de todos os subconjuntos finitos não vazios de $X$, então chamaremos $\left(X, \Omega_{X}\right)$ de um espaço de escolha finito.

Para qualquer espaço de escolha $\left(X, \Omega_{X}\right)$, uma correspondência de escolha em $\Omega_{X}$ é definida como qualquer correspondência $c: \Omega_{X} \rightrightarrows X$ tal que $\varnothing \neq c(A) \subseteq A$ para todo $A \in \Omega_{X}$. Note que esta definição contempla a possibilidade do tomador de decisão escolher mais de uma alternativa para qualquer problema de escolha $A \in \Omega_{X}$ com o qual ele se depare.

Por mais que esta seja uma formulação padrão, a interpretação precisa de mais de um elemento ser escolhido pode ser pouco clara, especialmente porque $X$ foi definido como um conjunto de alternativas "mutuamente exclusivas". Ademais, desde a criação da teoria da preferência revelada por Samuelson (1938), grande ênfase foi dada ao caráter observável da abordagem de escolha para a análise econômica, em oposição a abordagens baseadas no conceito de utilidade. Claramente, no entanto, não se pode observar um agente escolhendo muitas alternativas mutuamente exclusivas de uma vez, o que pode colocar em dúvida a formulação padrão.

Para maior concretude, interpretaremos escolhas múltiplas surgindo de $c(\cdot)$ como em Sen (1993), ao dizer que $c(A)$ é o conjunto de todos os elementos "escolhíveis" para um determinado problema de escolha $A$. Isto é, para qualquer problema de escolha arbitrário $A$, a decisão final do tomador de decisões será invariavelmente um membro de $c(A)$. O agente pode finalizar sua escolha ao randomizar subjetivamente dentre os membros de $A$.

Os seguintes postulados, que são frequentemente impostos em $c$, terão um papel fundamental neste artigo:

Axioma 1 (Chernoff - $\boldsymbol{\alpha}$ ) Para todo $A \in \Omega_{X}$, se $x \in c(A)$, então $x \in c(B)$ para todo $B \in \Omega_{X}$ tal que $x \in B \subseteq A$. 
O axioma $(\alpha)$ de Sen (1993) sugere que se uma alternativa é escolhida em algum problema de escolha, ela ainda será escolhida se o problema de escolha "encolher". Em particular, ele implica que $c(c(A))=c(A)$, supondo que $c(A)$ seja um problema de escolha. Foi originalmente proposto por Chernoff (1954).

Axioma 2 (Aizerman - AA) Se $A, B \in \Omega_{X}$ são tais que $c(A) \subseteq B \subseteq A$, então $c(B) \subseteq$ $c(A)$.

Este axioma, que teve seu papel fundamental na teoria da decisão, na escolha social e na teoria do controle reconhecida por Aizerman e Malishevski (1981), declara que eliminar algumas alternativas de um problema de escolha que não estão no conjunto escolhido não é capaz de fazer com que novas alternativas sejam escolhidas.

Axioma 3 (Expansão - $\gamma$ ) Para qualquer coleção $\mathcal{A} \subseteq \Omega_{X}$ tal que $\bigcup \mathcal{A}$ seja um problema de escolha, se $x \in c(A)$ para todo $A \in \mathcal{A}$, então $x \in c(\bigcup \mathcal{A})$.

Se alguma alternativa é escolhida ao longo de todos os problemas de escolha em uma coleção, então ela também deve ser escolhida na sua união, dado que esta seja em si mesma um problema de escolha.

\subsection{Preferências e categorias}

Como de costume, as partes simétrica e assimétrica de uma dada relação binária reflexiva $\succsim \subseteq X \times X$ serão denotadas por $\sim$ and $\succ$, respectivamente. Logo, $\succsim=\sim \cup \succ$, onde a união é disjunta. Vamos denotar por $\bowtie$ a parte incomparável de tal relação binária, ou seja, a relação irreflexiva e simétrica sobre $X$ definida por $x \bowtie y$ se, e somente se, $x \succsim y$ e $y \succsim x$ não são verdade. Com essa notação em mãos, a definição de $\Varangle$ segue de imediato. Além das propriedades usuais de relações binárias, nós definimos a seguinte:

Definição 1 (Comparabilidade Transitiva): Uma relação binária $\succsim \subseteq X \times X$ possui comparabilidade transitiva se $x \bowtie y$ e $y \bowtie z$ juntos implicam que $x \bowtie z$.

Qualquer relação binária transitiva e reflexiva sobre $X$ será chamada de relação de preferência sobre $X$. Alternativamente, podemos simplesmente nos utilizar do jargão matemático e chamar tal relação de uma preordem sobre $X$. Ambos os termos serão usados intercambiavelmente nessa dissertação. Para qualquer $S \subseteq X \mathrm{e} \succsim \subseteq X \times X$, o símbolo $\left.\succsim\right|_{S}$ denotará a restrição de $\succsim$ a $S$.

Seja $(Y, \succcurlyeq)$ qualquer conjunto preordenado, o que significa que $Y$ é um conjunto nãovazio arbitrário munido de alguma preordem $\succcurlyeq$. Para todo $T \subseteq Y$ definimos:

- $M A X(T, \succcurlyeq)=\{x \in T: \neg(y \succ x), \forall y \in T\}$, o conjunto de todos os elementos $\succcurlyeq$-maximais de $T$, e; 
- $\max (T, \succcurlyeq)=\{x \in T: x \succcurlyeq y, \forall y \in T\}$, o conjunto de todos os elementos $\succcurlyeq$-máximos de $T$.

Note que $\max (T, \succcurlyeq) \subseteq M A X(T, \succcurlyeq)$ e que os dois conjuntos são iguais se $\succcurlyeq$ é completa. Ademais, se $T$ é finito, $M A X(T, \succcurlyeq) \neq \varnothing$. Por outro lado, se $T$ for infinito, na ausência de qualquer requerimento adicional sobre $T$ ou $\succcurlyeq$, o conjunto de elementos $\succcurlyeq$-maximais pode muito bem ser vazio.

Dada uma coleção $\mathcal{S}$ de conjuntos quaisquer, dizemos ainda que $\mathcal{S}$ é uma cobertura de $X$ se $\bigcup \mathcal{S}=X$, ou seja, se a união de todos os $S \in \mathcal{S}$ "engloba" o conjunto $X$ inteiro.

Dado um conjunto preordenado $(X, \succsim)$, definimos uma categoria simplesmente como um subconjunto $S \subseteq X$ tal que $\succsim$ é completa em $X$. Mais formalmente:

Definição 2 (Categorização): Seja $(X, \succsim)$ um conjunto preordenado. Uma cobertura $\mathcal{S}$ de $X$ é uma categorização de $(X, \succsim)$ se $\left.\succsim\right|_{S}$ é completa para todo $S \in \mathcal{S}$. Chamaremos qualquer $S \in \mathcal{S}$ de uma categoria de $\mathcal{S}$.

Deve notar-se que a definição acima impõe muito pouca estrutura sobre a cobertura $\mathcal{S}$. Particularmente, ela não requer que as categorias sejam mutuamente disjuntas, nem mesmo que $\mathcal{S}$ seja uma cobertura maximal de $X$. Portanto, essa formulação permite que um indivíduo tenha \{comida chinesa\} como uma categoria, com \{macarrão chinês\} como uma categoria "à parte", mas inserida na primeira, e outra categoria, digamos \{macarrão\}, que também contenha \{macarrão chinês\} mas que não seja um subconjunto de $\{$ comida chinesa $\}$. Tais interseções entre categorias podem surgir se, por exemplo, múltiplos critérios forem usados no processo de categorização, e alguns desses critérios se sobreporem uns aos outros. ${ }^{1}$ Também vale ressaltar que não supomos que as categorias sejam necessariamente problemas de escolha. Em outras palavras, permitimos que a correspondência de escolha $c$ não seja definida sobre categorias, o significa que um agente pode não saber escolher entre todos os membros de uma categoria, se esta escolha lhe for apresentada.

Além disso, implícito na definição de categorização está o fato de que categorias são, pelo menos em parte, subjetivas. Isto é, a maneira pela qual o agente categoriza alternativas depende das suas preferências subjetivas. Portanto, não presumimos que categorias implícitas sejam observáveis, exceto talvez indiretamente, mediante o comportamento de escolha do tomador de decisões. Um observador externo não pode presumir, por exemplo, que $\{$ comida chinesa $\}$ e $\{$ comida mexicana $\}$ serão categorias relevantes para o tomador de decisão em qualquer aplicação específica. Isto está de acordo com estudos recentes no campo da psicologia cognitiva. ${ }^{2}$

1 Como Manzini e Mariotti (2012, pp. 11) exemplificam, "think of categorizing cameras by price band and brand, or flights by cheapness and convenience".

2 Por exemplo, Smith, Patalano e Jonides (1998) concluem que o método de categorização utilizado pelo indivíduo - se é baseado em regras ou em similaridades, por exemplo - impacta nas categorias resultantes de maneira previsível e significativa. 



\section{Escolha Sob Categorias}

\subsection{O caso geral}

Talvez a maneira mais natural de racionalizar escolhas na presença de categorias seja simplesmente escolher, dentre as alternativas apresentadas no problema de escolha, os melhores elementos de cada categoria. Para uma categorização $\mathcal{S} \subseteq 2^{X} \backslash\{\varnothing\}$ e uma relação de preferência $\succsim \subseteq X \times X$, podemos considerar que $c$ é racionalizado por uma maximização sob categorias se, para todo $A \in \Omega_{X}$,

$$
c(A)=\bigcup_{S \in \mathcal{S}} \max \left(A \cap S,\left.\succsim\right|_{S}\right) .
$$

Esse tipo de representação permite comportamentos que não seriam possíveis se a escolha fosse simplesmente o resultado de uma maximização de preferências incompletas sobre o problema de escolha, ou seja, $c(A)=M A X(A, \succsim)$ para todo $A \in \Omega_{X}$, como em Eliaz e Ok (2006). O exemplo a seguir ilustra esse ponto.

Exemplo 1 Seja $X=\{x, y, z\}$ e $\Omega_{X}=2^{X} \backslash\{\varnothing\}$. A correspondência de escolha definida por $c(\{x, y\})=\{x, y\}, c(\{x, z\})=\{x, z\}, c(\{y, z\})=\{y, z\}$ e $c(\{x, y, z\})=\{y, z\}$ ñ̃o pode ser racionalizada como $c(\cdot)=M A X(\cdot, \succsim)$, para nenhuma relação de preferência $\succsim$. De fato, c não satisfaz $(\gamma)$, o que, como veremos em breve, é uma condição necessária para tal representação.

Por outro lado, essa correspondência de escolha pode ser racionalizada por uma maximização sob categorias definindo $\succsim=\Delta \cup(y, x) \cup(z, x)^{1}$ e $\mathcal{S}=\{\{x, y\},\{x, z\}\}$.

Note que, no exemplo acima, pode ser que o elemento $x$ seja escolhido mesmo que todos os outros elementos de $X$ sejam estritamente preferidos a ele. Embora tal comportamento possa parecer contra-intuitivo à primeira vista, pode-se pensar nisso como uma predileção pela diversidade: pode ser que o indivíduo escolha uma opção preterida simplesmente porque ele esteja "com vontade" de consumir algo de uma certa categoria, mesmo que uma alternativa "melhor" de uma categoria diferente esteja disponível (um exemplo seria alguém que vai a um restaurante italiano e pede macarronada "para variar", mesmo que normalmente prefira comer pizza).

Tendo estabelecido que categorização é um mecanismo cognitivo importante, e que a análise de escolhas sob categorias não pode ser resumida à maximização de uma relação de preferência incompleta, resta determinar quais tipos de comportamento de escolha podem ser racionalizados por uma maximização sob categorias. Os resultados a seguir têm por objetivo responder essa pergunta no arcabouço mais geral considerado até agora, em que

$\overline{1}$ O símbolo $\Delta$ aqui representa o conjunto $\Delta:=\{(x, x) \in X \times X: x \in X\}$. 
a estrutura imposta sobre a categorização está restrita àquela da Definição 2. Começamos por enunciar um postulado.

Axioma 4 (Dominância) Existe uma relação binária acíclica $\succ$ tal que, para quaisquer $A, B \in \Omega_{X}$ com $A \subseteq B$, se $x \in c(A)$ mas $x \notin c(B)$, então existe $y \in B \backslash A$ com $y \succ x e$ $y \in c(A \cup\{y\})$.

Seja $\succsim$ qualquer preordem completa que estende $\succ$ (a existência de tal extensão foi estabelecida pela primeira vez por Szpilrajn (1930)). Podemos provar o lema a seguir:

Lema 1 Suponha que c: $\Omega_{X} \rightrightarrows X$ satisfaz $(\alpha)$ e Dominância. Para qualquer problema

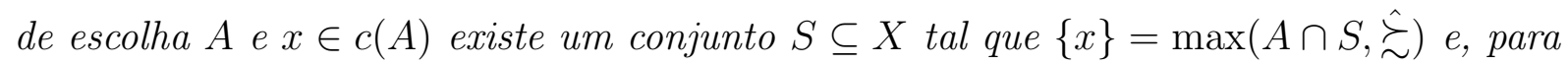
todo $B \in \Omega_{X}, \max (B \cap S, \hat{\succsim}) \subseteq c(B)$.

Demonstração. Fixe qualquer $A \in \Omega_{X}$ e $x \in c(A)$. Defina $S=\{x\} \cup\{y \in X \backslash A$ : $y \hat{\succsim} x$ e $y \in c(B)$ para todo $B \in \Omega_{X}$ com $\left.A \cup\{y\} \subseteq B \subseteq(A \cup\{z \in X: y \hat{\succsim} z\})\right\}$. Note que $A \cap S=\{x\}$ e, consequentemente, $\{x\}=\max (A \cap S, \hat{\succsim})$. Agora fixe qualquer problema de escolha $B$ e suponha que $y \in \max (B \cap S, \hat{\succsim})$. Precisamos da seguinte afirmação:

Afirmação 3.1.1 Para todo $z \in B \backslash A$ tal que ž̀y, nós temos que $z \notin c(A \cup\{z\})$.

Demonstração. Fixe $z \in B \backslash A$ tal que $z \hat{\succ} y$. Como, por construção, $y \in \max (B \cap S, \hat{\succsim})$, nós temos que $z \notin S$. Logo, existe um problema de escolha $E$ com $A \cup\{z\} \subseteq E \subseteq$ $(A \cup\{w \in X: z \hat{\succsim} w\})$ e $z \notin c(E)$. Mas então, se $z \in c(A \cup\{z\})$, deve existir $w \in X$ com $z \hat{\succsim} w$, mas $w \succ z$. Isso contradiz a construção de $\hat{\succsim}$.

Por causa de $(\alpha)$ e da afirmação acima, sabemos que $z \notin c(A \cup\{y, z\})$ para qualquer $z \in B \backslash A$ com $z \hat{\succ} y$. Mas então, Dominância implica que $y \in c(A \cup B)$. Por $(\alpha)$, isto implica que $y \in c(B)$. Concluimos $\max (B \cap S, \hat{\succsim}) \subseteq c(B)$.

O lema anterior permite que provemos o seguinte teorema de representação.

Teorema 1 Seja $\left(X, \Omega_{X}\right)$ um espaço de escolha arbitrário. Uma correspondência de escolha $c: \Omega_{X} \rightrightarrows X$ satisfaz $(\alpha)$ e Dominância se, e somente se, existe uma preordem

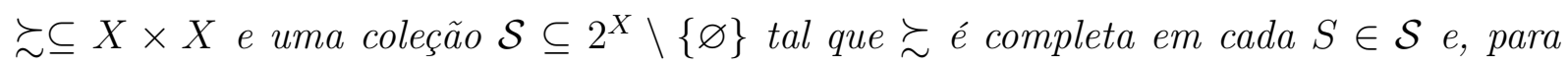
todo $A \in \Omega_{X}$,

$$
c(A)=\bigcup_{S \in \mathcal{S}} \max (A \cap S, \succsim)
$$

Demonstração. É fácil checar que a representação implica que c satisfaz os dois axiomas. Por outro lado, suponha que c satisfaz os dois axiomas e seja $\succsim$ uma preordem completa que estende a relação $\succ$. Pelo Lema 1, para todo problema de escolha A e cada $x \in c(A)$, existe $S_{x, A} \subseteq X$ tal que $\{x\}=\max \left(A \cap S_{x, A}, \succsim\right)$ e, para cada problema de escolha 
$B$ diferente de $A, \max \left(B \cap S_{x, A}, \succsim\right) \subseteq c(B)$. Seja $\mathcal{S}=\left\{S_{x, A} \subseteq X: x \in c(A)\right.$ e $\left.A \in \Omega_{X}\right\}$ a coleção de todos os conjuntos do tipo $S_{x, A}$. Então, para todo $A \in \Omega_{X}$,

$$
c(A)=\bigcup_{S \in \mathcal{S}} \max (A \cap S, \succsim) .
$$

O Teorema 1 estabelece as condições necessárias e suficientes para que uma correspondência de escolha seja racionalizável por maximização sob categorias. No entanto, ainda não chegamos exatamente ao nosso objetivo, já que o axioma de Dominância pressupõe a existência de uma relação acíclica $\succ$ com certas características desejáveis. Idealmente, deveríamos extrair tal relação binária diretamente da correspondência de escolha.

Como candidata para a relação que precisamos, defina $P$ por $x P y$ se, e somente se, existe um problema de escolha $A$ tal que $y \in c(A)$ mas $y \notin c(A \cup\{x\})$. Impomos o seguinte postulado sobre $c$ :

Axioma 5 (Aciclicidade) A relação $P$ é acíclica.

Quando consideramos espaços de escolha finitos, o próximo lema é o último ingrediente necessário para a caracterização que buscamos.

Lema 2 Seja $\left(X, \Omega_{X}\right)$ um espaço de escolha finito e c : $\Omega_{X} \rightrightarrows X$ uma correspondência de escolha sobre $\Omega_{X}$ que satisfaça $(\alpha)$, AA e Aciclicidade. Então c satisfaz Dominância com $P$ como a relação binária relevante.

Demonstração. Tome quaisquer $A, B \in \Omega_{X}$ com $A \subseteq B$ tais que $x \in c(A)$ e $x \notin c(B)$. Note primeiro que $c(B) \backslash A \neq \varnothing$. Com efeito, suponha que $c(B) \subseteq A$. Então $A A$ implica que $c(A) \subseteq c(B)$, o que contradiz o fato de que $x \notin c(B)$. Por $(\alpha)$, se $y \in c(B)$ então $y \in c(A \cup\{y\})$. Portanto, basta mostrar que existe $y \in c(B) \backslash A$ tal que yPx.

Agora, $(\alpha)$ e $A A$ implicam que $c(A \cup B)=c(A \cup c(B)){ }^{2}$ Suponha, buscando uma contradição, que yPx seja falso para todo $y \in c(B)$. Logo, $x \in c(A \cup\{y\})$ para todo $y \in c(B)$. Lembre-se que $c(B)$ é finito e seja $\left\{y_{1}, \ldots, y_{n}\right\}$ uma enumeração arbitrária de $c(B)$. Como $y_{1} P x$ não é verdadeiro, temos que $x \in c\left(A \cup\left\{y_{1}\right\}\right)$. Por indução, suponha que $x \in c\left(A \cup\left\{y_{1}, \ldots, y_{m-1}\right\}\right)$ e considere $c\left(A \cup\left\{y_{1}, \ldots, y_{m-1}\right\} \cup\left\{y_{m}\right\}\right)$. Como $y_{m} P x$ é falso, devemos ter $x \in c\left(A \cup\left\{y_{1}, \ldots, y_{m}\right\}\right)$ para todo $1 \leq m \leq n$. Portanto, $x \in$ $c\left(A \cup\left\{y_{1}, \ldots, y_{n}\right\}\right)=c(A \cup c(B))$, uma contradição.

Concluimos que para qualquer par de problemas de escolha $A$ e $B$, com $A \subseteq B$, tais que $x \in c(A)$, mas $x \notin c(B)$, existe $y \in c(B) \backslash A \subseteq B \backslash A$ com $y \in c(A \cup\{y\})$.

Agora estamos prontos para enunciar o principal resultado deste trabalho, que nos dá uma caracterização completa, para espaços de escolha finitos, dos tipos de correspondências de escolha que são racionalizáveis por uma maximização sujeita a uma dada categorização. Sua prova segue-se diretamente do Teorema 1 e do Lema 2.

2 Esta propriedade é denominada Path Independence. Ver, por exemplo, Moulin (1985). 
Teorema 2 Seja $\left(X, \Omega_{X}\right)$ um espaço de escolha finito. Uma correspondência de escolha $c: \Omega_{X} \rightrightarrows X$ satisfaz $(\alpha), A A$ e Aciclicidade se, e somente se, existe uma preordem $\succsim \subseteq X \times X$ e uma coleção de conjuntos $\mathcal{S} \subseteq 2^{X} \backslash\{\varnothing\}$ tais que $\succsim$ é completa em cada $S \in \mathcal{S}$ e, para todo $A \in \Omega_{X}$,

$$
c(A)=\bigcup_{S \in \mathcal{S}} \max \left(A \cap S,\left.\succsim\right|_{S}\right)
$$

\subsection{Categorias disjuntas}

Uma forma de categorização que desperta particular interesse, dada a sua ubiquidade, é quando as categorias são mutuamente disjuntas. Com efeito, em psicologia e filosofia, a visão clássica de categorização, que se remete a Aristóteles, afirma que categorias são entidades discretas, plenamente caracterizadas por condições necessárias e suficientes para adesão. ${ }^{3}$ Portanto, de acordo com esse ponto de vista, categorias são, por definição, mutuamente exclusivas. ${ }^{4}$

Embora nosso interesse primordial não seja endorsar qualquer teoria filosófica ou psicológica em particular, o próprio fato da visão clássica ter sido, por tanto tempo, o paradigma dominante no estudo de categorização indica que, de alguma maneira, ela seja um jeito "natural" de se pensar sobre categorias. Assim sendo, devotamos esta seção a caracterizar um teorema de representação para quando a categorização é uma partição de $X$.

Como ficará claro a seguir, a representação por maximização sob categorias disjuntas está intimamente relacionada com a maximização de preferências incompletas, no estilo de Eliaz e Ok (2006). Mas à medida que o caso geral, apresentado anteriormente, é menos restritivo do que simplesmente maximizar preferências incompletas, o caso com categorias disjuntas impõe mais restrições. O seguinte resultado estabelece este fato e nos dá o ingrediente-chave para encontrar a caracterização que buscamos.

Lema 3 Seja $\left(X, \Omega_{X}\right)$ um espaço de escolha arbitrário e c : $\Omega_{X} \rightrightarrows X$ qualquer correspondência de escolha. Existe uma relação de preferência $\succsim$ sobre $X$ que satisfaz Comparabilidade Transitiva tal que, para todo $A \in \Omega_{X}$,

$$
c(A)=\operatorname{MAX}(A, \succsim)
$$

se, e somente se, existe uma partição $\mathcal{S}$ de $X$ e uma relação de preferência $\succsim$ tal que para todo $S \in \mathcal{S},\left.\succsim\right|_{S}$ é completa sobre $S$ e, para todo $A \in \Omega_{X}$,

$$
c(A)=\bigcup_{S \in \mathcal{S}} \max \left(A \cap S,\left.\succsim\right|_{S}\right)
$$

3 Para uma revisão de teorias de categorização na psicologia, veja por exemplo Smith e Medin (1981) e Komatsu (1992).

4 Na verdade, o único tipo de interseção entre categorias permitido pela visão clássica é quando uma categoria é um subconjunto, ou refinamento, de outra. Portanto, pode-se dizer que, para o mesmo grau de generalidade, as categorias são disjuntas. 
Demonstração. Primeiro assuma que existe uma preordem $\succsim$ sobre $X$ satisfazendo Comparabilidade Transitiva tal que, para todo $A \in \Omega_{X}, c(A)=M A X(A, \succsim)$. Para cada $x \in X$, seja $\mathcal{S}_{x}=\left\{S \subseteq X: x \in S\right.$ e $\left.\succsim\right|_{S}$ é uma preordem completa $\}$. Note que, como $\succsim$ é reflexiva, $\mathcal{S}_{x} \neq \varnothing$ para todo $x \in X$.

Agora fixe $x \in X$ e seja $\left(\mathcal{S}_{x}, \supseteq\right)$ um conjunto preordenado. Seja $\mathcal{S}_{x}^{\prime}$ qualquer subconjunto totalmente ordenado de $\mathcal{S}_{x}$. É fácil ver que o conjunto $\bigcup \mathcal{S}_{x}^{\prime}$ está contido em $\mathcal{S}_{x}$ e representa um limite superior para $\mathcal{S}_{x}^{\prime}$. O Lema de Zorn agora implica que $\operatorname{MAX}\left(\mathcal{S}_{x}, \supseteq\right) \neq$ $\varnothing$ para todo $\mathcal{S}_{x} \subseteq 2^{X} \backslash\{\varnothing\}$. Por construção, $\left.\succsim\right|_{S}$ é completa em todo $S \in \operatorname{MAX}\left(\mathcal{S}_{x}, \supseteq\right)$.

Portanto defina $\mathcal{S}=\left\{M A X\left(\mathcal{S}_{x}, \supseteq\right) \in 2^{X} \backslash\{\varnothing\}: x \in X\right\}$. Pelo argumento acima, $\mathcal{S} \neq \varnothing$. Ademais, como $x \in S$ para todo $S \in M A X\left(\mathcal{S}_{x}, \supseteq\right)$, para todo $x \in X$, temos que $\cup \mathcal{S}=X$. Procederemos demonstrando algumas afirmações.

Afirmação 3.2.1 Para todo $S \in \mathcal{S}$, se $y \in X \backslash S$, então $\exists x \in S$ tal que $y \bowtie x$.

Demonstração. Fixe $S \in \mathcal{S}$ e tome $y \in X \backslash S$. Se y pode ser comparado a todo $x \in S$, então $S \cup\{y\} \supset S$ é completamente ordenado por $\succsim$, o que contradiz a $\supseteq$-maximalidade de $S$.

Afirmação 3.2.2 $\mathcal{S}$ é uma partição de $X$.

Demonstração. Por construção, $\bigcup \mathcal{S}=X$. Resta provar que $\forall S, T \in \mathcal{S}$ tais que $S \neq T$, temos necessariamente $S \cap T=\varnothing$.

Fixe $S, T \in \mathcal{S}, S \neq T$, e suponha que $S \cap T \neq \varnothing$. Tome $x \in S \cap T$. Por construção, $x$ é comparável a qualquer $y \in S$ e qualquer $z \in T$. Como comparabilidade é transitiva, devemos ter $y \succsim z$ ou $z \succsim y$, para todo $(y, z) \in S \times T$. Portanto $\left.\succsim\right|_{S \cup T}$ é completa. Como $S$ e $T$ são ambos conjuntos maximais na ordem de inclusão (já que eles pertencem a $\mathcal{S}$ ), temos necessariamente que $S, T \subset S \cup T$. Mas isso contradiz a própria maximalidade de $S$ e T. Logo, $S \cap T=\varnothing$.

Afirmação 3.2.3 Para todo $A \in \Omega_{X}, \operatorname{MAX}(A, \succsim) \subseteq \bigcup_{S \in \mathcal{S}} \max \left(A \cap S,\left.\succsim\right|_{S}\right)$.

Demonstração. Fixe $A \in \Omega_{X}$. Tome qualquer $x \in M A X(A, \succsim)$, o que implica que $x \bowtie y$ ou $x \succsim y$ para todo $y \in A$. Como $\bigcup \mathcal{S}=X$, existe algum $\bar{S} \in \mathcal{S}$ tal que $x \in \bar{S}$ e, além disso, $\left.x \succsim\right|_{\bar{S}}$ y ou $\left.y \succsim\right|_{\bar{S}} x$ para todo $y \in \bar{S}$. Portanto, para qualquer $z \in A \cap \bar{S}$ temos $\left.x \succsim\right|_{\bar{S}} z$, o que implica que $x \in \max \left(A \cap \bar{S},\left.\succsim\right|_{\bar{S}}\right)$.

Afirmação 3.2.4 Para todo $A \in \Omega_{X}, \bigcup_{S \in \mathcal{S}} \max \left(A \cap S,\left.\succsim\right|_{S}\right) \subseteq M A X(A, \succsim)$.

Demonstração. Fixe qualquer $A \in \Omega_{X}$ e $x \in \bigcup_{S \in \mathcal{S}} \max \left(A \cap S,\left.\succsim\right|_{S}\right)$. Claramente, $\exists \hat{S} \in \mathcal{S}$ tal que $x \in \max \left(A \cap \hat{S},\left.\succsim\right|_{\hat{S}}\right)$. Suponha que $x \notin M A X(A, \succsim)$. Então existe $y \in A$ com $y \succ x$. Por definição de $\max \left(A \cap \hat{S},\left.\succsim\right|_{\hat{S}}\right)$, obtemos $y \notin A \cap \hat{S}$, o que implica, devido à Afirmação 3.2.1, que $\exists z \in \hat{S}$ tal que $z \bowtie y$. Como $x$ é comparável a todo 
$w \in \hat{S}$ e comparabilidade é transitiva, temos em particular que y é comparável a z, uma contradição.

Logo, $\forall A \in \Omega_{X}$,

$$
c(A)=M A X(A, \succsim)=\bigcup_{S \in \mathcal{S}} \max \left(A \cap S,\left.\succsim\right|_{S}\right)
$$

Seja c : $\Omega_{X} \rightrightarrows X$ uma correspondência de escolha e assuma agora que existe uma partição $\mathcal{S}$ de $X$ e uma relação de preferência $\succsim$ tal que para todo $S \in \mathcal{S},\left.\succsim\right|_{S}$ é completa sobre $S$ e, para todo $A \in \Omega_{X}$,

$$
c(A)=\bigcup_{S \in \mathcal{S}} \max \left(A \cap S,\left.\succsim\right|_{S}\right)
$$

Para todo $x, y \in X$, defina a relação binária $\succeq$ como

$$
x \succeq y \Longleftrightarrow x, y \in S \in \mathcal{S} \text { e }\left.x \succsim\right|_{S} y
$$

Afirmação 3.2.5 $\succeq$ é uma preordem.

Demonstração. Obviamente $\succeq$ é reflexiva. Para demonstrar que é transitiva, tome $x, y, z \in X \operatorname{com} x \succeq y$ e $y \succeq z$. Então $x, y \in S$ e $y, z \in T,\left.\operatorname{com} x \succsim\right|_{S}$ y e $\left.y \succsim\right|_{T} z$, para algum $S, T \in \mathcal{S}$. Como $\mathcal{S}$ é uma partição, deve ser o caso que $S=T$, pois de outro modo $S \neq T$ e $y \in S \cap T \neq \varnothing$, uma contradição. Logo, $x, z$ estão na mesma categoria. Ademais, como $\left.\right|_{S \cup T}$ é transitiva, $\left.x \succsim\right|_{S \cup T} z$. Portanto, $x \succeq z$.

Afirmação 3.2.6 $\succeq$ satisfaz Comparabilidade Transitiva.

Demonstração. Tome quaisquer $x, y, z \in X$ tais que $x \succeq y$ e $z \succeq y$. Então o mesmo argumento da afirmação anterior garante que $x$ e z estão na mesma categoria. Agora, completude das preferências dentro das categorias implica que $x$ é comparável a z.

Afirmação 3.2.7 Para todo $A \in \Omega_{X}, c(A)=M A X(A, \succeq)$.

Demonstração. Primeiramente fixe $x \in c(A)$. Então $x \in \max \left(A \cap S,\left.\succsim\right|_{S}\right)$ para algum $S \in \mathcal{S}$. Suponha que $x \notin M A X(A, \succeq)$. Então $\exists y \in A$ tal que $y \succ x$. Logo, $x, y \in A \cap S$ e $\left.y \succ\right|_{S} x$, o que contradiz o fato de que $x$ é um máximo em $A \cap S$. Portanto, $c(A) \subseteq$ $\operatorname{MAX}(A, \succeq)$.

Agora tome $x \in M A X(A, \succeq)$, o que significa que não existe $y \in A$ tal que $y \succ$ x. Em particular, $\nexists z \in A \cap S$ tal que $\left.z \succ\right|_{S} x$. Logo, $x \in M A X\left(A \cap S,\left.\succsim\right|_{S}\right)$. Como completude de $\succsim$ sobre $S$ implica que $\operatorname{MAX}\left(A \cap S,\left.\succsim\right|_{S}\right)=\max \left(A \cap S,\left.\succsim\right|_{S}\right)$, temos que $x \in \max \left(A \cap S,\left.\succsim\right|_{S}\right)$. Portanto, $\operatorname{MAX}(A, \succeq) \subseteq c(A)$. 
Nós demonstramos que

$$
c(A)=\bigcup_{S \in \mathcal{S}} \max \left(A \cap S,\left.\succsim\right|_{S}\right)=\operatorname{MAX}(A, \succeq)
$$

onde $\succeq$ é uma relação de preferência que satisfaz Comparabilidade Transitiva.

Acabamos de estabelecer que a representação com categorias disjuntas é um caso especial da maximização de preferências incompletas. No intuito de usar este fato para fornecer uma caracterização completa da nossa representação, seria útil determinar primeiro sob quais condições uma correspondência de escolha satisfaz $c(\cdot)=M A X(\cdot, \succsim)$ para alguma preordem $\succsim$. O próximo teorema, devido a Riella e Ribeiro (2015), faz exatamente isso. Antes de apresentar este teorema, no entanto, definiremos dois postulados.

Axioma 6 (WWARNI) Para todo $A \in \Omega_{X}$ e $y \in A$, se para todo $x \in A$ existe um conjunto $B_{x} \in \Omega_{X}$ com $y \in c\left(B_{x}\right)$ e $x \in B_{x}$, então $y \in c(A)$.

WWARNI foi proposto pela primeira vez por Riella e Ribeiro (2015), que mostraram sua equivalência a $(\alpha)$ conjuntamente com $(\gamma)$.

Axioma 7 (Transitividade das Escolhas Estritas - TEE) Para todo $\{x, y, z\} \subseteq X$, se $\{x\}=c(\{x, y\}) e\{y\}=c(\{y, z\})$, então $\{x\}=c(\{x, z\})$.

Teorema 3 (Riella e Ribeiro (2015)) Seja $\left(X, \Omega_{X}\right)$ um espaço de escolha e $c: \Omega_{X} \rightrightarrows$ $X$ uma correspondência de escolha. Então, c satisfaz WWARNI e TEE se, e somente se, existe uma relação de preferência $\succsim$ sobre $X$ tal que $c(A)=M A X(A, \succsim)$ para todo $A \in \Omega_{X}$.

A questão agora se resume a gerar Comparabilidade Transitiva na representação impondo algum axioma diretamente sobre a correspondência de escolha. A propriedade abaixo vem a ser exatamente o que precisamos.

Axioma 8 (Comparabilidade Mútua Revelada - CMR) Para todo $A \in \Omega_{X}$ tal que exista $x \in X$ com $|c(\{x, y\})|=1$ para cada $y \in A$, nós temos $c(B) \cap A=c(B \cap A)$, para todo $B \in \Omega_{X}$ tal que $c(B) \cap A \neq \varnothing$.

O axioma CMR afirma que se um dado problema de escolha $A$ é tal que existe um $x \in X$ tal que todas as escolhas binárias entre $x$ e elementos de $A$ são estritas, então todas as alternativas de $A$ são, em certo sentido, reveladas comparáveis umas às outras. Além de gerar Comparabilidade Transitiva, na presença do axioma $(\alpha)$ temos que CMR engloba TEE, como iremos demonstrar.

Lema 4 Se c : $\Omega_{X} \rightrightarrows X$ satisfaz $(\alpha)$ e CMR, então também satisfaz TEE. 
Demonstração. Tome quaisquer $x, y, z \in X$ tais que $\{x\}=c(\{x, y\}) e\{y\}=c(\{y, z\})$. Aplicando $(\alpha)$ duas vezes, nós obtemos $\{x\}=c(\{x, y, z\})$. Agora, como $|c(\{x, y\})|=$ $|c(\{y, z\})|=1$, por CMR temos que $c(\{x, y, z\}) \cap\{x, z\}=c(\{x, y, z\} \cap\{x, z\})=c(\{x, z\})$. Isto implica que $\{x\}=c(\{x, z\})$.

Portanto, a discussão que se segue prescinde completamente de TEE. O próximo lema é de crucial importância para o nosso propósito.

Lema 5 Seja $\left(X, \Omega_{X}\right)$ um espaço de escolha e c : $\Omega_{X} \rightrightarrows X$ qualquer correspondência de escolha sobre $\Omega_{X}$. Então, c satisfaz $(\alpha),(\gamma)$ e CMR se, e somente se, existe uma relação de preferência $\succsim$ sobre $X$ que satisfaz Comparabilidade Transitiva tal que, para todo $A \in \Omega_{X}, c(A)=M A X(A, \succsim)$.

Demonstração. Suponha primeiro que c(.) satisfaz $(\alpha),(\gamma)$ e CMR, e defina a parte assimétrica da relação binária $\succsim$ da seguinte forma, para todo $x, y \in X$ :

$$
x \succ y \Longleftrightarrow x \neq y \text { e c }(\{x, y\})=\{x\}
$$

Seja $\mathcal{P}(c)=\{(x, y) \in X \times X: x \neq y$ e c $(\{x, y\})=\{x, y\}\}$ e $\mathcal{C}(c)=\{(x, y) \in \mathcal{P}(c):$ $\exists z \in X$ com $|c(\{x, z\})|=|c(\{y, z\})|=1\}$. Agora defina a parte simétrica de $\succsim$ como

$$
x \sim y \Longleftrightarrow x=y \text { ou }(x, y) \in \mathcal{C}(c)
$$

Claramente, $\sim$ é reflexiva e disjunta de $\succ$. Portanto, defina $\succsim=\succ \cup \sim$ e note que $\succsim e ́$ obviamente reflexiva.

Afirmação 3.2.8 $c(A)=M A X(A, \succsim), \forall A \in \Omega_{X}$.

Demonstração. Suponha que $x \in c(A)$ para algum $A \in \Omega_{X}$, mas que $y \succ x$ para algum $y \in A$. Pela definição de $\succsim$, esta última afirmação implica que $\{y\}=c(\{x, y\})$, o que contradiz $(\alpha)$. Logo, $c(A) \subseteq M A X(A, \succsim)$, para qualquer $A \in \Omega_{X}$ escolhido arbitrariamente. Para provar que $M A X(\cdot, \succsim) \subseteq c(\cdot)$, pegue qualquer $A \in \Omega_{X}$, tome $x \in M A X(A, \succsim) e$ assuma que $x \notin c(A)$. Por $W W A R N I$, existe algum $y \in A$ tal que $x \notin c\left(B_{y}\right)$ para todo $B_{y} \in \Omega_{X}$ com $y \in B_{y}$. Em especial, $\{y\}=c(\{x, y\})$, ou seja, $y \succ x$, o que contradiz $x \in M A X(A, \succsim)$. Concluímos que $c(A)=\operatorname{MAX}(A, \succsim)$ para todo $A \in \Omega_{X}$.

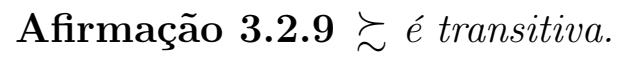

Demonstração. Tome quaisquer $x, y, z \in X$ distintos, com $x \succsim y \succsim z$. Se $x \succ y \succ z$, por definição $\{x\}=c(\{x, y\})$ e $\{y\}=c(\{y, z\})$, então segue-se a partir de $(\alpha)$ e CMR que $\{x\}=c(\{x, z\})$. Note que, tendo em vista este último resultado, para quaisquer $x, y \in X$ tais que $x \sim y$, existe $z \in X \operatorname{com} c(\{x, z\})=\{x\}$ e $c(\{y, z\})=\{y\}$, ou com $c(\{x, z\})=c(\{y, z\})=\{z\}$. 
Se $x \succ y \sim z$, então $(\alpha)$ implica que $y \notin c(\{x, y, z\})$, e portanto $c(\{x, y, z\}) \cap\{y, z\} \neq$ $\{y, z\}$. Logo, CMR e $(y, z) \in \mathcal{C}(c)$ implicam que $c(\{x, y, z\}) \cap\{y, z\}=\varnothing$, o que por sua vez implica que $c(\{x, y, z\})=\{x\}$, então por $(\gamma)$ obtemos $c(\{x, z\})=\{x\}$.

Assuma agora que $x \sim y \succ z$. Então claramente $z \succ x e z \sim x$ são falsos, pois qualquer um desses implicaria que $y \succ x$. Suponha portanto, buscando uma contradição, que $(x, z) \in \mathcal{P}(c) \backslash \mathcal{C}(c)$. Se existir $w \in X$ com $c(\{w, x\})=c(\{w, y\})=\{w\}$, temos que $w \succ y \succ z$, então $w \succ z$, e portanto $w \in X$ é tal que $c(\{x, w\})=c(\{z, w\})=\{w\}$. Portanto, $x \sim z$, o que já mostramos ser falso. Se $w$ é tal que $c(\{x, w\})=\{x\} e$ $c(\{y, w\})=\{y\}$, então se $c(\{z, w\})=\{z, w\}$ teríamos $z \sim w$, já que $y \in X$ seria tal que $y \succ z$ e $y \succ w$. Logo, decorreria dos resultados do parágrafo anterior que $x \succ z$. Se $c(\{z, w\})=\{w\}$, transitividade de $\succ$ também implicaria que $x \succ z$. Finalmente, se $c(\{z, w\})=\{z\}$, então $c(\{x, z\})=\{x, z\}$ implica que $x \sim z$, uma contradição.

Finalmente, considere o caso em que $x \sim y \sim z$, com $x, y$ e $z$ distintos. Pelo argumento acima, evidentemente $x \succ z$ ou $z \succ x$ não se sustentam, então necessariamente temos $(x, z) \in \mathcal{P}(c)$. Queremos mostrar que $\exists w \in X$ com $|c(\{x, w\})|=|c(\{z, w\})|=1$. Como $y \sim z$, existe $v \in X$ tal que $y, z \succ v$ ou $v \succ y, z$. Se $y \succ v$ for o caso, então $x \sim y \succ v$ implica que $x \succ v$, então $x, z \succ v$. Argumento completamente análogo vale para $v \succ y$. Colocando $w=v$, obtemos $x \sim z$.

Afirmação 3.2.10 $\succsim$ satisfaz Comparabilidade Transitiva.

Demonstração. Para provar que $\succsim$ demonstra transitividade da comparabilidade, tome quaisquer $x, y, z \in X$ distintos, com $x \succ y$ e $z \succ y$. Então se $|c(\{x, z\})|=1$, não há nada para demonstrar. Se, por outro lado, $\{x, z\} \in \mathcal{P}(c)$, nós temos que $|c(\{x, y\})|=$ $|c(\{z, y\})|=1$, e portanto $x \sim z$.

Para provar a outra parte do resultado, seja $\succsim$ uma relação de preferência sobre $X$ que satisfaz Comparabilidade Transitiva e suponha que $c(\cdot)=M A X(\cdot, \succsim)$. Desejamos demonstrar que c satisfaz $(\alpha),(\gamma)$ e CMR.

Afirmação 3.2.11 c satisfaz $C M R$.

Demonstração. Fixe um conjunto $A \in \Omega_{X}$ qualquer tal que exista algum $x \in X$ com $|c(\{x, y\})|=|\operatorname{MAX}(\{x, y\}, \succsim)|=1$ para todo $y \in A$ e considere qualquer $B \in \Omega_{X}$ tal que $c(B) \cap A \neq \varnothing$. Como $\succsim$ possui comparabilidade transitiva, por construção temos que para todo $y, z \in A, y$ e z são comparáveis.

Suponha que $y \notin M A X(B \cap A, \succsim)$. Então existe $x \in B \cap A$ com $x \succ y$, o que implica que $x \in B$ e $x \succ y$, logo $y \notin M A X(B, \succsim) \supseteq M A X(B, \succsim) \cap A$. Portanto $M A X(B, \succsim) \cap A \subseteq M A X(B \cap A, \succsim)$.

Agora pegue $y \in M A X(B \cap A, \succsim)$ e suponha, buscando uma contradição, que $y \notin$ $M A X(B, \succsim) \cap A$. Como $y \in A$, devemos ter $y \notin M A X(B, \succsim)$. Logo, se existe $x \in A \cap B$ 
com $x \succ y$, então $y \notin M A X(B \cap A, \succsim)$, uma contradição. Portanto, é necessariamente verdade que $x \notin A$ para todo $x \in M A X(B, \succsim)$ tal que $x \succ y$. Além disso, qualquer

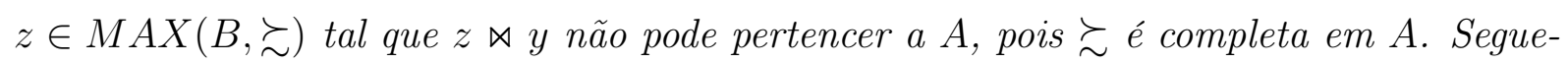
se que $M A X(B, \succsim) \cap A=\varnothing$, o que também leva a uma contradição. Portanto, $M A X(B \cap$ $A, \succsim) \subseteq M A X(B, \succsim) \cap A$, e concluimos que, $\forall B \in \Omega_{X} \operatorname{com} c(B) \cap A \neq \varnothing$,

$$
c(B) \cap A=c(B \cap A)
$$

Finalmente para uma demonstração de que c satisfaz $(\alpha)$ e $(\gamma)$, ver Riella e Ribeiro (2015, Teorema 1).

Podemos agora resumir todos os resultados desta seção num único teorema elegante, que nos proporciona uma caracterização completa de escolhas racionais sob categorias disjuntas.

Teorema 4 Seja $\left(X, \Omega_{X}\right)$ um espaço de escolha arbitrário e c : $\Omega_{X} \rightrightarrows X$ qualquer correspondência de escolha sobre $\Omega_{X}$. Então, c satisfaz WWARNI e CMR se, e somente se, existe uma partição $\mathcal{S}$ de $X$ e uma relação de preferência $\succsim$ tal que para todo $S \in \mathcal{S},\left.\succsim\right|_{S}$ é completa sobre $S$ e, para todo $A \in \Omega_{X}$,

$$
c(A)=\bigcup_{S \in \mathcal{S}} \max \left(A \cap S,\left.\succsim\right|_{S}\right)
$$

Demonstração. Segue-se diretamente dos lemas 3 e 5. 


\section{Conclusão}

Nós apresentamos um modelo puro de comportamento de escolhas dentro de categorias. Categorização é um mecanismo cognitivo universal, e sua importância para a cognição e tomada de decisões dos seres humanos é amplamente reconhecida em muitas áreas, como psicologia, filosofia e linguística.

Recentemente, alguns pesquisadores em economia também têm se interessado por este fenômeno, em particular Mullainathan, Schwartzstein e Shleifer (2008) e Manzini e Mariotti (2012). Contudo, os modelos destes são bastante diferentes do nosso. Mullainathan, Schwartzstein e Shleifer (2008) focam nos efeitos de categorizações - ou, nos termos deles, "coarse thinking" - para o processo de atualização bayesiana, e aplicam o modelo ao estudo de persuasão não-informativa. Por outro lado, Manzini e Mariotti (2012) desenvolvem uma teoria pura da escolha na presença de categorização, mas assumem que o indivíduo escolhe tanto entre categorias quanto dentro das categorias.

A representação exposta neste artigo provê uma descrição precisa das escolhas dentro de categorias, mas se abstém de analisar preferências entre elas. Este método tem duas grandes vantagens: (i) do ponto de vista empírico, sabe-se que comparações entre categorias são complicadas, e às vezes impossíveis, e (ii) ela é capaz de levar em consideração algumas das maiores violações da racionalidade plena, como certas formas de efeitos de menu (menu effects). Ao mesmo tempo que deixamos o método de escolha entre categorias como uma questão em aberto, encorajamos pesquisas futuras sobre o assunto.

Ademais, no quadro mais geral considerado na seção 3.1, nós tomamos categorias como dadas e impomos pouquíssimas restrições sobre suas possíveis estruturas. Isto permite que nosso modelo explique uma ampla variedade de padrões comportamentais. no entanto, ele não diz nada sobre que tipos de procedimentos de categorização são efetivamente tomados em situações concretas. Apesar de que determinar o processo através do qual categorias são formadas é uma questão importante e interessante, isto está além do escopo desta dissertação. É um tema de valor para empreitadas futuras. 



\section{Referências}

AIZERMAN, M.; MALISHEVSKI, a. General theory of best variants choice: Some aspects. IEEE Transactions on Automatic Control, v. 26, n. 5, p. 1030-1040, 1981. ISSN 0018-9286.

CHERNOFF, H. Rational selection of decision functions. Econometrica: Journal of the Econometric Society, v. 22, n. 4, p. 422-443, 1954. Disponível em: <http: //www.jstor.org/stable/10.2307/1907435>.

ELIAZ, K.; OK, E. Indifference or indecisiveness? Choice-theoretic foundations of incomplete preferences. Games and Economic Behavior, v. 56, n. 1, p. 61-86, jul. 2006. ISSN 08998256. Disponível em: <http://linkinghub.elsevier.com/retrieve/pii/ S0899825606000169>.

KOMATSU, L. K. Recent views of conceptual structure. Psychological Bulletin, v. 112, n. 3, p. 500-526, 1992. ISSN 0033-2909.

MANZINI, P.; MARIOTTI, M. Categorize then choose: Boundedly rational choice and welfare. Journal of the European Economic Association, v. 10, p. 1141-1165, 2012. ISSN 15424766.

MEDIN, D. L.; AGUILAR, C. Categorization. [S.l.]: MIT Press, 1999.

MOULIN, H. Choice functions over a finite set: A summary. Social Choice and Welfare, v. 2, p. 147-160, 1985. ISSN 01761714.

MUllainATHAN, S.; SCHWARTZSTEIN, J.; SHLEIFER, A. Coarse Thinking and Persuasion. The Quarterly Journal of Economics, v. 123, n. 2, p. 577-619, 2008. ISSN 00335533. Disponível em: < http://www.jstor.org/stable/25098910>.

RIELLA, G.; RIBEIRO, M. On rational choice with incomplete preferences. 2015.

SAMUELSON, P. A note on the pure theory of consumer's behaviour: an addendum. Economica, v. 5, n. 17, p. 61-71, 1938. ISSN 00130427. Disponível em: $<$ http://www.jstor.org/stable/2548634\$ delimiter"026E30F\$npapers2://publication/ uuid/4308E4F4-3BCC-4710-A7A2-CB85E3A78FC7>.

SCHWARTZ, B. Self-determination. The tyranny of freedom. The American psychologist, v. 55, n. 1, p. 79-88, 2000. ISSN 0003-066X.

SEN, A. Internal Consistency of Choice. 1993. 495-521 p. Disponível em: < http: //www.ams.org/leavingmsn?url=http://dx.doi.org/10.2307/2951715>.

SMITH, E.; MEDIN, D. Categories and concepts. 1981. 203 p. Disponível em: <http://www.cs.indiana.edu/ $\sim$ port/teach/sem08/Smith.Medin.1983.ch1.2.3.pdf $>$.

SMITH, E. E.; PATALANO, a. L.; JONIDES, J. Alternative strategies of categorization. Cognition, v. 65, p. 167-196, 1998. ISSN 00100277.

SZPILRAJN, E. Sur l'extension de l'ordre partiel. Fundamenta Mathematicae, v. 16, n. 1, p. 386-389, 1930. ISSN 0016-2736. 